\title{
Primordial Nucleosynthesis
}

\author{
Alain Coc* \\ Centre de Sciences Nucléaires et de Sciences de la Matière (CSNSM), CNRS/IN2P3, \\ Université Paris Sud 11, UMR 8609, Bâtiment 104, F-91405 Orsay Campus (France) \\ E-mail: cocecsnsm.in2p3.fr
}

\section{Elisabeth Vangioni}

Institut d'Astrophysique de Paris, UMR-7095 du CNRS, Université Pierre et Marie Curie, 98 bis bd Arago, 75014 Paris (France)

Sorbonne Universités, Institut Lagrange de Paris, 98 bis bd Arago, 75014 Paris (France).

E-mail: vangioni@iap.fr

Primordial nucleosynthesis, or big bang nucleosynthesis (BBN), is one of the three evidences for the big bang model, together with the expansion of the Universe and the Cosmic Microwave Background. There is a good global agreement over a range of nine orders of magnitude between abundances of ${ }^{4} \mathrm{He}, \mathrm{D},{ }^{3} \mathrm{He}$ and ${ }^{7} \mathrm{Li}$ deduced from observations, and calculated in primordial nucleosynthesis. However, there remain, a yet-unexplained, discrepancy of a factor $\approx 3$, between the calculated and observed lithium primordial abundances, that has not been reduced, neither by recent nuclear physics experiments, nor by new observations. The precision in Deuterium observations in cosmological clouds has recently improved dramatically, so that nuclear cross sections involved in Deuterium BBN need to be known with similar precision. We will shortly discuss nuclear aspects related to $\mathrm{BBN}$ of $\mathrm{Li}$ and $\mathrm{D}, \mathrm{BBN}$ with non-standard neutron sources, and finally, improved sensitivity studies using Monte Carlo that can be used in other site of nucleosynthesis.

XIII Nuclei in the Cosmos,

7-11 July, 2014

Debrecen, Hungary

\footnotetext{
*Speaker.
} 


\section{Introduction}

Besides the universal expansion, the Cosmic Microwave Background (CMB) radiation the third evidence for the big bang model comes from primordial or big bang Nucleosynthesis (BBN). During the first $\approx 20$ minutes of the universe, when it was dense and hot enough for nuclear reactions to take place, BBN produced "light elements", ${ }^{4} \mathrm{He}, \mathrm{D},{ }^{3} \mathrm{He}$ and ${ }^{7} \mathrm{Li}$, together with minute traces of ${ }^{6} \mathrm{Li},{ }^{9} \mathrm{Be},{ }^{11} \mathrm{~B}$ and $\mathrm{CNO}$.

The nuclear reaction rates affecting the production of the $A<8$ isotopes have all been measured in nuclear physics laboratories or can be calculated from the standard theory of weak interactions. In that case, they are normalized to the experimental value for the lifetime of the neutron. Its precise value is still a matter of debate [34], but its uncertainty has only marginal effect on BBN. After the number of light neutrino families, the last parameter to have been independently determined is the precise value of baryonic density of the Universe, which is now deduced from the observations of the anisotropies of the CMB radiation. It is usual to introduce $\eta$, the number of photons per baryon which remains constant during the expansion, (after electron-positron annihilation) and is directly related to $\Omega_{\mathrm{b}}$ (the baryonic density relative to the critical density) by $\Omega_{\mathrm{b}} \cdot h^{2}=3.6521 \times 10^{7} \eta$ [9] with

$$
\Omega_{\mathrm{b}} h^{2}=0.02218 \pm 0.00026
$$

according to the first release of the Planck mission collaboration ("Planck+lensing+WP+highL") [1] ( $h$ represents the Hubble constant $\left(H_{0}\right)$ in units of $100 \mathrm{~km} / \mathrm{s} / \mathrm{Mpc}$ ).

The number of free parameters in Standard Big Bang Nucleosynthesis has now been reduced to zero, and the calculated primordial abundances are in principle only affected by the moderate uncertainties in some nuclear cross-sections. These primordial abundances can be compared with astronomical observations in primitive astrophysical sites. Figure 2 and Table 1 in Ref. [33] show that even though the agreement with observations is good or very good for ${ }^{4} \mathrm{He},{ }^{3} \mathrm{He}$ and $\mathrm{D}$, there is a tantalizing discrepancy for ${ }^{7} \mathrm{Li}$ that has not yet found a consensual explanation.

Hence, present big bang nucleosynthesis studies are focused on $i$ ) solving the Lithium problem, ii) improving the accuracy of the predictions to match increasing precision on observations and iii) probe the physics of the early universe. Indeed, when we look back in time, it is the ultimate process for which, a priori, we know all the physics involved: departure from its predictions could provide hints or constraints on new physics or astrophysics [21,27].

\section{The light elements: ${ }^{4} \mathrm{He}, \mathrm{D},{ }^{3} \mathrm{He}$ and ${ }^{7} \mathrm{Li}$}

We refer to Ref. [33] for a comparison between BBN predictions and observations, using updated baryonic density, neutron lifetime and an extended nuclear network. However, we will discuss briefly here the nuclear aspects related to the Lithium problem, and to the increased precision on $\mathrm{D} / \mathrm{H}$ observations.

There are $\approx 12$ nuclear reactions responsible for the production of ${ }^{4} \mathrm{He}, \mathrm{D},{ }^{3} \mathrm{He}$ and ${ }^{7} \mathrm{Li}$ in Standard BBN. There are many other reactions connecting these isotopes, but their cross sections are too small and/or reactants too scarce to have any significant effect. Even among these 12 reactions, a few of them (e.g. ${ }^{3} \mathrm{H}(\mathrm{d}, \mathrm{n}){ }^{4} \mathrm{He}$ and $\left.{ }^{3} \mathrm{H}(\alpha, \gamma){ }^{7} \mathrm{Li}\right)$ are now irrelevant at $\mathrm{CMB}$ deduced 
baryonic density. Unlike in other sectors of nuclear astrophysics, those nuclear cross sections have usually been directly measured at BBN energies [15] (a few $100 \mathrm{keV}$ ). The weak reactions involved in $\mathrm{n} \leftrightarrow \mathrm{p}$ equilibrium are an exception: their rates come from the standard theory of the weak interaction, normalized to the experimental neutron lifetime. Its precise value is still a matter of debate [34], but awaiting further experimental progress, we adopt the recommended value of $\tau_{\mathrm{n}}$ $=880.1 \pm 1.1 \mathrm{~s} \mathrm{[4]}$ (see its influence in Ref. [9]).

\subsection{The lithium problem}

In spite of new laboratory measurements, there is still a factor of $\approx 3$ between the calculated $\left[\mathrm{Li} / \mathrm{H}=\left(4.94_{-0.40}^{+0.38}\right) \times 10^{-10}[9]\right]$ and the one deduced from observations $\left[\mathrm{Li} / \mathrm{H}=\left(1.58_{-0.28}^{+0.35}\right) \times 10^{-10}\right.$ $[32]],{ }^{7} \mathrm{Li}$ primordial abundances. Before invoking non-standard solutions to this large discrepancy (see Fields [17] for a summary of proposed solutions and e.g. Yamazaki et al. [35] for a recently proposed model), nuclear solutions need to be ruled out.

At the baryonic density deduced from $\mathrm{CMB}$ observations, ${ }^{7} \mathrm{Li}$ is produced indirectly by ${ }^{3} \mathrm{He}(\alpha, \gamma){ }^{7} \mathrm{Be}$, that will, much later decay to ${ }^{7} \mathrm{Li}$ while it is destroyed by ${ }^{7} \mathrm{Be}(\mathrm{n}, \mathrm{p}){ }^{7} \mathrm{Li}(\mathrm{p}, \alpha){ }^{4} \mathrm{He}$. The ${ }^{3} \mathrm{He}(\alpha, \gamma)^{7} \mathrm{Be}$ cross-section has long been a subject of debate because of systematic differences that were found according to the experimental technique: prompt or activation measurements. Fortunately, new experiments have allowed Cyburt \& Davids [12] to calculated an improved S-factor, with reduced uncertainty ${ }^{1}$.

To solve this problem, within conventional nuclear physics, is to search for other reactions that could lead to ${ }^{7} \mathrm{Li}+{ }^{7} \mathrm{Be}$ increased destruction. The ${ }^{7} \mathrm{Be}(\mathrm{d}, \mathrm{p}) 2 \alpha$ reaction was a prime candidate but subsequent experiments and analyses ruled out this possibility ([23] and references therein). Extending this search, recent works [5] suggested the possibility of overlooked resonances in nuclear reactions involving ${ }^{7} \mathrm{Be}$, the most promising candidate was found to be in the ${ }^{7} \mathrm{Be}+{ }^{3} \mathrm{He} \rightarrow{ }^{10} \mathrm{C}$ channel. Indeed, the presence of a level close to the ${ }^{7} \mathrm{Be}+{ }^{3} \mathrm{He}$ reaction threshold $(\mathrm{Q}=15.003 \mathrm{MeV})$ in ${ }^{10} \mathrm{C}$ [5], with favorable properties would help alleviate the lithium problem. However, a recent experiment was conducted at the Tandem of the Orsay ALTO facility to improve the ${ }^{10} \mathrm{C}$ and ${ }^{11} \mathrm{C}$ spectroscopy. The ${ }^{10} \mathrm{~B}\left({ }^{3} \mathrm{He}, \mathrm{t}\right){ }^{10} \mathrm{C}$ and ${ }^{11} \mathrm{~B}\left({ }^{3} \mathrm{He}, \mathrm{t}\right){ }^{11} \mathrm{C}$ reactions were investigated at a ${ }^{3} \mathrm{He}$ beam energy of $35 \mathrm{MeV}$ and the tritons analyzed by the Split-pole magnet. Only upper limits for the presence of new levels in ${ }^{10} \mathrm{C}$ and ${ }^{11} \mathrm{C}$ were obtained, too low to have an impact on ${ }^{7} \mathrm{Li}$ production [19].

An other nuclear physics scenario requires an increased late time neutron abundance rendering the ${ }^{7} \operatorname{Be}(n, p){ }^{7} \operatorname{Li}(\mathrm{p}, \alpha)^{4} \mathrm{He}$ channel more efficient (see $\S 3$ ).

\subsection{Deuterium}

The deuterium abundance closest to primordial abundance is determined from the observation of very few clouds at high redshift (Fig. 1), on the line of sight of distant quasars. Recently, Cooke et al. [10] have made new observations and reanalysis of existing data, that lead to a new average value of $\mathrm{D} / \mathrm{H}=(2.53 \pm 0.04) \times 10^{-5}$, lower and with smaller uncertainties than in previous determinations. Deuterium BBN predictions are marginally compatible with BBN predictions of

\footnotetext{
${ }^{1}$ After this Conference, an improved evaluation of the ${ }^{3} \mathrm{He}(\alpha, \gamma){ }^{7} \mathrm{Be}$ reaction rate and associated uncertainty has been published [14].
} 


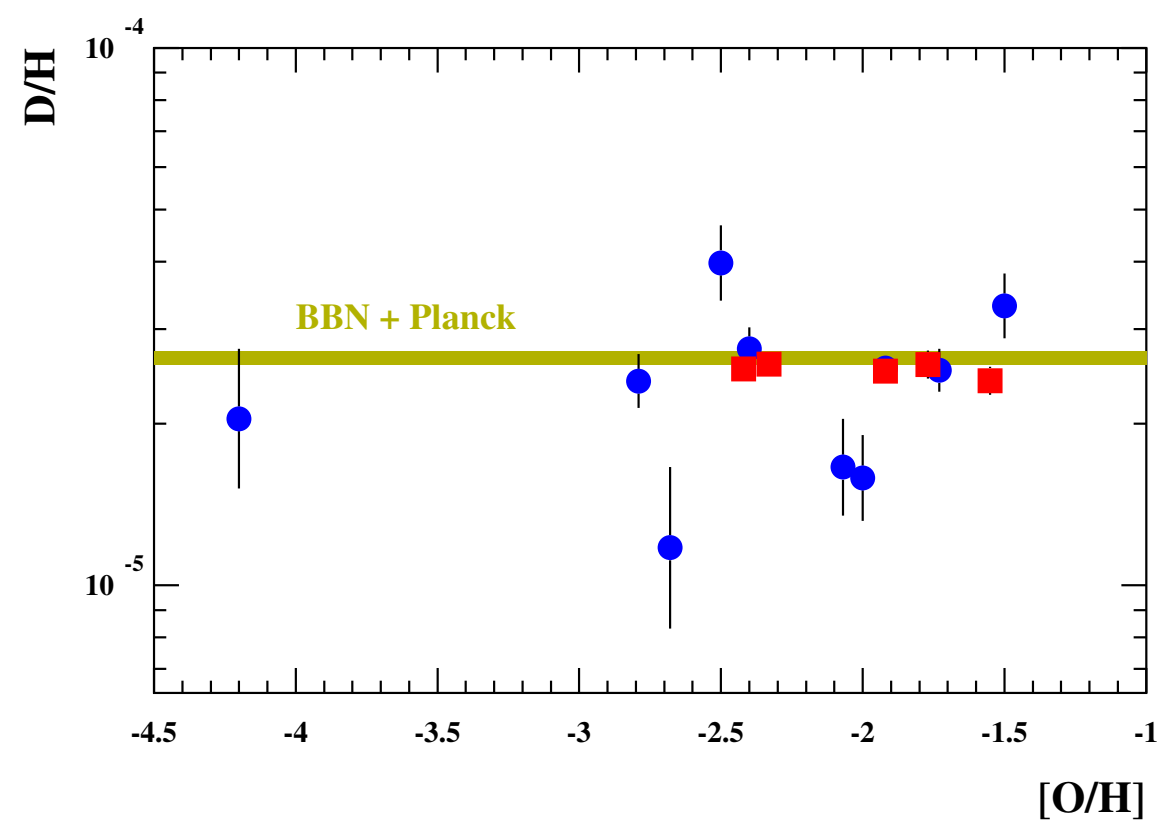

Figure 1: D/H observations, as a function of metallicity, from Pettini et al. [30] (blue circles) and Cooke et al. [10] (red squares). These most recent observations [10] have very small error bars and show very few dispersion, and are just slightly below BBN calculations [9, 33].

$\mathrm{D} / \mathrm{H}=(2.656 \pm 0.067) \times 10^{-5}[1],(2.54 \pm 0.17) \times 10^{-5}[28,13]$ and $\left(2.64_{-0.07}^{+0.08}\right) \times 10^{-5}[9,33]$. If such a precision of $1.6 \%$ in observations is confirmed, great care should be paid to nuclear cross sections affecting Deuterium nucleosynthesis.

Sensitivity studies (e.g. Ref. [11, 6]) have shown that the ${ }^{2} \mathrm{H}(\mathrm{d}, \mathrm{n}){ }^{3} \mathrm{He},{ }^{2} \mathrm{H}(\mathrm{d}, \mathrm{p})^{3} \mathrm{H}$ and ${ }^{2} \mathrm{H}(\mathrm{p}, \gamma)^{3} \mathrm{H}$ reactions, are the most influential on $\mathrm{D} / \mathrm{H}$ predicted abundance: a $10 \%$ variation to their rates induces a relative variation of respectively $-5.5 \%,-4.6 \%$ and $-3.2 \%$ on $\mathrm{D} / \mathrm{H}$. Concerning these reactions, since the last dedicated $\mathrm{BBN}$ evaluations of reaction rates $[11,31,15]$ a new experiment was performed by Leonard et al. [25]. They measured both the ${ }^{2} \mathrm{H}(\mathrm{d}, \mathrm{n})^{3} \mathrm{He},{ }^{2} \mathrm{H}(\mathrm{d}, \mathrm{p})^{3} \mathrm{H}$ cross section between $\approx 50-300 \mathrm{MeV}$, i.e. well within $\mathrm{BBN}$ energy range, with a quoted uncertainty of $2 \% \pm 1 \%$. On the contrary, no new experiment concerning the ${ }^{2} \mathrm{H}(\mathrm{p}, \gamma)^{3} \mathrm{H}$ reaction has been conducted so that its rate uncertainty $(5 \%-8 \%[15,2])$, according to Di Valentino et al. [16], now dominates the error budget on $\mathrm{D} / \mathrm{H}$ predictions.

\section{Non standard neutron injection during BBN}

It was recognized (e.g. Jedamzik [22]), that extra neutron injection would increase ${ }^{7} \mathrm{Be}$ destruction by ${ }^{7} \mathrm{Be}(\mathrm{n}, \mathrm{p})^{7} \mathrm{Li}(\mathrm{p}, \alpha)^{4} \mathrm{He}$, but at the expense of a rise in the abundance of $\mathrm{D} / \mathrm{H}$. Given the new tight constraints [10], one may question if the neutron injection mechanism is still a valid agent for reducing the cosmological abundance of lithium. Extending the BBN network to $\approx 400$ reactions has not lead to the identification of any overlooked conventional neutron source i.e. an 
extra neutron producing reaction. Hence, one has to investigate non standard neutron sources that can be:

1. Particle decay. This class of models assumes the existence of an hypothetical particle $X$ that can decay and produce neutron, i.e. $X \rightarrow \mathrm{n}+\ldots \ldots$

2. Particle annihilation. These models assumes $X+X \rightarrow \mathrm{n}+\ldots$. pair annihilation.

3. Resonant particle annihilation. A narrow resonance in the annihilation cross section is present at some energy.

4. $n-n^{\prime}$ oscillation. This model [3] assumes that there is a mirror world from which mirrorneutrons can oscillate into our world. The microphysics is considered to be identical in the two sectors, but the temperatures and baryonic densities are different in the two sectors [3].

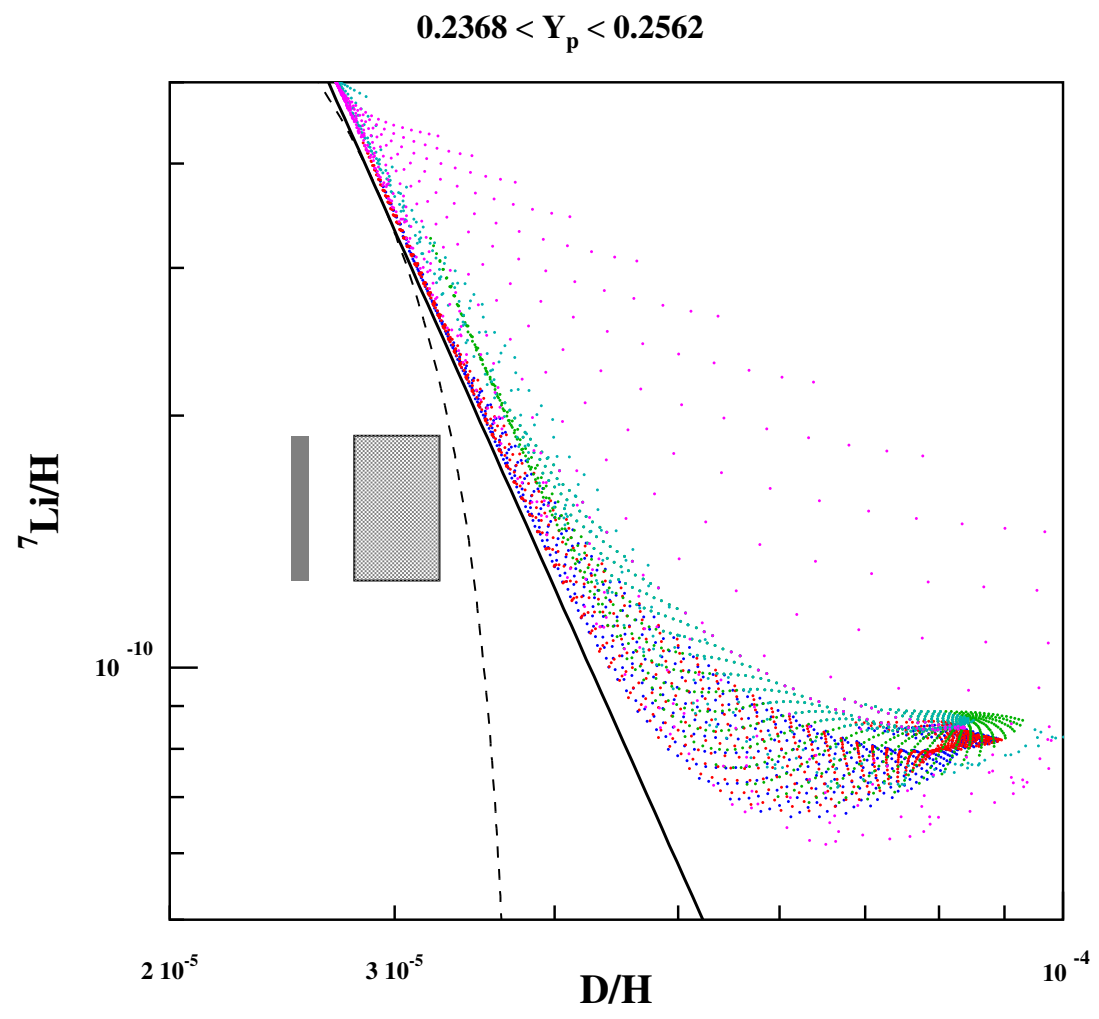

Figure 2: Each dot is the prediction of a model $[8]$ in the space $\left(\mathrm{D} / \mathrm{H},{ }^{7} \mathrm{Li} / \mathrm{H}\right)$. The left/right rectangle corresponds to the D/H data of Ref. [10]/Ref. [28]. The lithium abundance corresponds to the value of Ref. [32]. This demonstrates that no model can be in agreement with both lithium-7 and deuterium. The blue, red and green dots correspond to $n-n$ ' oscillation models the light blue dots correspond to resonant annihilation models and the pink dots to particle decay models. The solid line corresponds to Eq. 3.1 and the dashed to the limit for instantaneous neutron injection from Kusakabe et al. [24]. 
Figure 2 is a summary of the results [8] of BBN calculations within the framework of models $2-4$, while varying the relevant parameters. Each dot correspond to a set of parameters and different colors correspond to different models [8]. It is easily concluded that all the models lies on the halfplane above the dashed line, that is empirically:

$$
\log (\mathrm{D} / \mathrm{H})>-0.293 \log \left({ }^{7} \mathrm{Li} / \mathrm{H}\right)-7.3 .
$$

It is clear, of course, that the ${ }^{7} \mathrm{Be}$ destruction by the injection of extra neutrons is accompanied by the deuterium production due to the $n+p$ channel. One can see that along this line, lithium and deuterium abundances are indeed anti-corellated but that along this line the lithium abundance is more sensitive to the neutron injection that deuterium. This is indeed a common feature of neutron injection models e.g. instantaneous injection [24] lead to the limiting curve shown as a dashed line in Fig. 2, or following massive gravitino decay (Fig. 1 in Ref. [28]).

\section{Improved sensitivity studies using Monte Carlo and CNO production}

The Monte Carlo technique is now widely used in nucleosynthesis calculations [18]. Ideally reaction rate uncertainties are known, together with the associated probability density functions (p.d.f.). As described in Longland et al. [26], this can be obtained by Monte Carlo calculations taking into account uncertainties and p.d.f. of experimentally (or theoretically) determined quantities that enter into the rate calculations. Reaction rate p.d.f. can usually be represented by a log-normal distribution whose parameters are tabulated as a function of temperature [26].

These Reaction rate p.d.f. can then be used in nucleosynthesis Monte Carlo calculations where all reaction rates are sampled independently. From the resulting histograms of calculated abundances, the median and $68 \%$ confidence interval is obtained from the $0.5,0.16$ and 0.84 quantiles. This is how the $(68 \%)$ confidence intervals quoted here are obtained e.g. the CNO Standard Big Bang Nucleosynthesis production is found to be $\mathrm{CNO} / \mathrm{H}\left(0.96_{-0.47}^{+1.89}\right) \times 10^{-15}$ (too low to have an impact on Population III stellar evolution) [9]. In a simple sensitivity study [7] (i.e. varying one reaction rate at a time), unpredicted effect were found, e.g. increasing the ${ }^{7} \mathrm{Li}(\mathrm{d}, \mathrm{n}) 2^{4} \mathrm{He}$ reaction rate reduces the $\mathrm{CNO}$ abundance, even though the ${ }^{4} \mathrm{He}, \mathrm{D},{ }^{3} \mathrm{He}$ and ${ }^{7} \mathrm{Li}$ final abundances are left unchanged.

However, Monte Carlo results can be used for more detailed analyses. For instance, Fig. 4 of Ref. [9], shows that the CNO abundance distribution is not gaussian and that in $\approx 2 \%$ of the cases, $\mathrm{CNO} / \mathrm{H}>10^{-13}$, a value that may affect Pop. III stellar evolution. Calculating correlation coefficients [18] between CNO final abundance and reaction rates, it has been possible to identify four reactions, involving ${ }^{10} \mathrm{Be}$, responsible for this effect. The combination of higher rates for ${ }^{10} \mathrm{Be}(\alpha, \mathrm{n}){ }^{13} \mathrm{C}$ and ${ }^{8} \mathrm{Li}(\mathrm{t}, \mathrm{n}){ }^{10} \mathrm{Be}$ together with lower rates for ${ }^{10} \mathrm{Be}(\mathrm{p}, \alpha){ }^{7} \mathrm{Li}$ and ${ }^{10} \mathrm{Be}(\mathrm{p}, \mathrm{t}) 2^{4} \mathrm{He}$ result in a substantial increase in primordial CNO production [9]. Note that, the previous simple sensitivity study was not able to identify reactions that could induce such an effect: changing each of these reaction rate, one at a time, by factors up to 1000 did not produce a change in excess of $30 \%$ for CNO abundance [7].

This demonstrate the importance of sensitivity studies in nuclear astrophysics, that in its simplest form can display unexpected effects, e.g. the influence of the ${ }^{7} \mathrm{Li}(\mathrm{d}, \mathrm{n}) 2^{4} \mathrm{He}$ reaction on $\mathrm{CNO}$ and in a more elaborate form (analysis of correlations) can identify the effect of chains of reactions. 


\section{Conclusion}

The agreement between BBN predictions and observations is quite satisfactory except for Lithium. Many studies have been devoted to the resolution of this Lithium problem and many possible "solutions", none fully satisfactory, have been proposed. For a detailed analysis see [17] and the various contributions to the meeting "Lithium in the cosmos" [36]. In particular nuclear physics solutions, leading to an increased ${ }^{7} \mathrm{Be}$ destruction, have been experimentally investigated, and can now be excluded [19]. Even though, they cannot, in any way, provide a solution, a better precision on reaction rates for ${ }^{3} \mathrm{He}(\alpha, \gamma){ }^{7} \mathrm{Be}$ and sub-]eading processes like ${ }^{7} \mathrm{Be}(\mathrm{n}, \alpha){ }^{4} \mathrm{He}$ and ${ }^{7} \mathrm{Be}(\mathrm{d}, \mathrm{p}) 2{ }^{4} \mathrm{He}$ would allow to improve the prediction of ${ }^{7} \mathrm{Li}$ primordial abundance. Now that the D/H primordial abundance is expected to be known with an improved precision [10], nuclear cross sections of all reactions leading to D destruction should be determined with an equal precision [16]. Finally, it cannot be excluded that, through a chain of reactions involving ${ }^{10} \mathrm{Be}$, a significantly increased primordial CNO production could be achieved.

\section{Acknowledgments}

We are indebted to our collaborators on these topics: Pierre Descouvemont, Stéphane Goriely, Keith Olive, Maxim Pospelov and Jean-Philippe Uzan. This work made in the ILP LABEX (under reference ANR-10-LABX-63) was supported by French state funds managed by the ANR within the Investissements d'Avenir programme under reference ANR-11-IDEX-0004-02 and by the ANR VACOUL, ANR-10-BLAN-0510.

\section{References}

[1] Planck Collaboration XVI, P. A. R. Ade, N. Aghanim et al., Planck 2013 results. XVI. Cosmological parameters, [arXiv:1303.5076].

[2] E. G. Adelberger, A. García, R. G. Hamish Robertson, et al., Solar fusion cross sections. II. The pp chain and CNO cycles Rev. Mod. Phys. 83 (2011) 195.

[3] Z. Berezhiani, D. Comelli, and F.L. Villante, The early mirror universe: inflation, baryogenesis, nucleosynthesis and dark matter, Phys. Lett. B 503 (2001) 362.

[4] J. Beringer et al., (Particle Data Group), The Review of Particle Physics, Phys. Rev. D 86 (2012) 010001 .

[5] N. Chakraborty, B. D. Fields and K. A. Olive, Resonant Destruction as a Possible Solution to the Cosmological Lithium Problem,' Phys. Rev. D 83 (2011) 063006.

[6] A. Coc, E. Vangioni, Big-Bang Nucleosynthesis with updated nuclear data, J. of Physics Conference Series 202, 012001 (2010).

[7] A. Coc, S. Goriely, Y. Xu, M. Saimpert \& E. Vangioni, Standard big bang nucleosynthesis up to CNO with an improved extended nuclear network, Astrophys. J. 744 (2012) 158.

[8] A. Coc, M. Pospelov, J.-Ph. Uzan and E. Vangioni Modified big bang nucleosynthesis with non-standard neutron sources Phys. Rev. D in press [arXiv:1405.1718] 
[9] A. Coc, J.-Ph. Uzan and E. Vangioni Standard big bang nucleosynthesis and primordial CNO Abundances after Planck, Journal of Cosmology and Astroparticle Physics, in press [arXiv:1403.6694].

[10] R. Cooke, M. Pettini, R. A. Jorgenson, M. T. Murphy \& C. C. Steidel, Precision measures of the primordial abundance of deuterium, Astrophys. J. 781 (2014) 31.

[11] R.H. Cyburt, Primordial nucleosynthesis for the new cosmology: Determining uncertainties and examining concordance, Phys. Rev. D 70, 023505 (2004).

[12] R. H. Cyburt \& B. Davids, Evaluation of modern ${ }^{3} H e(\alpha, \gamma){ }^{7}$ Be data, Phys. Rev. C 78 (2008) 064614.

[13] R.H. Cyburt, B.D. Fields, and K.A Olive, An update on the big bang nucleosynthesis prediction for ${ }^{7} \mathrm{Li}$ : the problem worsens Journal of Cosmology and Astroparticle Physics 11 (2008) 012

[14] R. J. deBoer, J. Görres, K. Smith, E. Uberseder, M. Wiescher, A. Kontos, G. Imbriani, A. Di Leva and F. Strieder, Monte Carlo uncertainty of the $\left.{ }^{3} \mathrm{He}(\alpha, \gamma)\right)^{7}$ Be reaction rate, Phys. Rev. $\mathrm{C} 90$ (2014) 035804.

[15] P. Descouvemont, A. Adahchour, C. Angulo, A. Coc \& E. Vangioni-Flam, Compilation and R-matrix analysis of Big Bang nuclear reaction rates, Atomic Data and Nuclear Data Tables 88 (2004) 203, http: //pntpm.ulb.ac.be/bigbang/.

[16] E. Di Valentino, C. Gustavino, J. Lesgourgues et al. Probing nuclear rates with Planck and BICEP2, Phys. Rev. D 90 (2014) 023543.

[17] B. D. Fields, The Primordial Lithium Problem, ARNPS 6 (2011) 47.

[18] C. Iliadis, R. Longland, A. Coc, F.X. Timmes \& A. E. Champagne, Statistical methods for thermonuclear reaction rates and nucleosynthesis simulations J. Phys. G, in press [arXiv:1409.5541].

[19] F. Hammache, A. Coc, N. de Séréville, I. Stefan, P. Roussel et al. Search for new resonant states in ${ }^{10} \mathrm{C}$ and ${ }^{11} \mathrm{C}$ and their impact on the cosmological lithium problem Phys. Rev. C88 (2013) 062802(R) [arXiv:1312.0894].

[20] F. Iocco, G. Mangano, G. Miele, O. Pisanti, P.D. Serpico, Path to metallicity: Synthesis of CNO elements in standard BBN, Phys. Rev. D 75, 7304 (2007).

[21] F. Iocco, G. Mangano, G. Miele, O. Pisanti \& P.D. Serpico, Primordial nucleosynthesis: From precision cosmology to fundamental physics, Phys. Rep. 472 (2009) 1.

[22] K. Jedamzik, Did something decay, evaporate, or annihilate during Big Bang nucleosynthesis?, Phys. Rev. D 70 (2004) 063524.

[23] O. S. Kirsebom and B. Davids, One fewer solution to the cosmological lithium problem, Phys. Rev. C 84 (2011) 058801.

[24] M. Kusakabe, M.-K. Cheoun and K. S. Kim, General limit on the relation between abundances of D and ${ }^{7}$ Li in big bang nucleosynthesis with nucleon injections, Phys. Rev. D 90 (2014) 045009.

[25] D.S. Leonard, H.J. Karwowski, C.R. Brune, B.M. Fisher and E.J. Ludwig, Precision measurements of ${ }^{2} H(d, p)^{3} H$ and ${ }^{2} H(d, n)^{3}$ He total cross sections at Big Bang nucleosynthesis energies, Phys. Rev. $\mathrm{C} 73$ (2006) 045801

[26] R. Longland, C. Iliadis, A. E. Champagne et al., Charged-particle thermonuclear reaction rates: I. Monte Carlo method and statistical distributions, Nucl. Phys. A 841 (2010) 1. 
[27] G. Mathews, Big Bang nucleosynthesis and the origin and evolution of space-time, these proceedings.

[28] K.A. Olive, P. Petitjean, E. Vangioni, J. Silk, Higher D or Li: probes of physics beyond the standard model, Mon. Not. R. Astron. Soc. 426, 1427 (2012).

[29] M. Paris, G. Hale, A. Hayes-Sterbenz, and G. Jungman, $R$-matrix analysis of reactions in the ${ }^{9} B$ compound system, Nuclear Data Sheets, 120 (2014) 184.

[30] M. Pettini \& M. Cooke, A new, precise measurement of the primordial abundance of deuterium, Mon. Not. R. Astron. Soc. 425 (2012) 2477.

[31] P. D. Serpico, S. Esposito, F. Iocco, G. Mangano, G. Miele and O. Pisanti, Nuclear reaction network for primordial nucleosynthesis: a detailed analysis of rates, uncertainties and light nuclei yields, Journal of Cosmology and Astroparticle Physics 12 (2004) 010.

[32] L. Sbordone, P. Bonifacio, E. Caffau et al., The metal-poor end of the Spite plateau I. Stellar parameters, metallicities, and lithium abundances, Astron. Astrophys. 522 (2010) A26.

[33] E. Vangioni and A. Coc, Updating Standard Big-Bang Nucleosynthesis after Planck, these proceedings.

[34] F. Wietfeldt and G. Greene, The neutron lifetime, Rev. Mod. Phys. 83 (2011) 1173.

[35] D. G. Yamazaki, M. Kusakabe, T. Kajino, G.J. Mathews and M.K. Cheoun, Cosmological solutions to the lithium problem: Big-bang nucleosynthesis with photon cooling, X-particle decay and a primordial magnetic field, Phys. Rev. D 90 (2014) 023001.

[36] Proceedings of the workshop Lithium in the cosmos, Mem.S.A.It. Suppl. 22 (2012), Paris, February 27-29, 2012, Editors: F. Iocco, P. Bonifacio, E. Vangioni. 